\title{
A Clinical Report of the Tooth-Implant Associated Double Crown Denture by Placing Additional Implants
}

\author{
Jun-Yeon Kim, DDS, MS', Jin-Hyun Cho, DDS, MS, PhD ${ }^{2 *}$ \\ ${ }^{1}$ Resident, Department of Prosthodontics, School of Dentistry, Kyungpook National University, Daegu, Korea \\ ${ }^{2}$ Professor, Department of Prosthodontics, School of Dentistry, Kyungpook National University, Daegu, Korea \\ *Corresponding author: Jin-Hyun Cho, Department of Prosthodontics, School of Dentistry, Kyungpook \\ National University, 2175, Dalgubeoldae-ro, Jung-gu, Daegu 41940, Korea \\ Tel : +82-53-600-7651. Fax : +82-53-427-0778. E-mail : prosth95@knu.ac.kr
}

pISSN 2765-7833

eISSN 2765-7841

Journal of implantology and applied sciences 2021; 25(4): 179-187

https://doi.org/10.32542/implantology.2021019

Received: September 28, 2021

Revised: October 14, 2021

Accepted: October 16, 2021

ORCID

Jun-Yeon Kim

http://orcid.org/0000-0002-3975-3558

Copyright (C) 2021. The Korean Academy of Oral \& Maxillofacial Implantology

This is an Open Access article distributed under the terms of the Creative Commons Attribution Non-Commercial License (http://creativecommons. org/licenses/by-nc/4.0/) which permits unrestricted non-commercial use, distribution, and reproduction in any medium, provided the original work is properly cited.

\section{Abstract}

When partially edentulous patients are treated using removable partial dentures, the teeth used as abutments are critical for prosthesis success. However, for patients with few remaining teeth, the prognosis of such abutment teeth supporting the clasp of removable partial dentures is of concern. In contrast, several studies have reported the success of treatments using double-crown prostheses for patients with few remaining teeth as periodontally compromised teeth can be used as abutments for double-crown dentures even when their prognosis is doubtful for removable partial dentures with clasps. This provides more options for treatment planning for partially edentulous patients with few remaining teeth. In this study, the patient was treated using a conus double-crown denture, where the inner and outer crowns were fabricated using a gold alloy. After 12 years, a new prosthesis was planned due to loss of denture retention and extraction of an abutment tooth. For the new prosthesis, three additional implants were placed and the original abutment teeth with gold inner crowns, except for the one that was extracted, were maintained and were used as abutments for the new hybrid telescopic double crown denture using a friction pin method. Although the opposing arch had natural teeth and fixed prostheses, the patient adapted well after denture delivery and was overall satisfied.

Keywords: Combined tooth/implant-supported removable prostheses, Dental implant, Double crown, Friction pin, Implant-borne restorations

\section{I . Introduction}

The use of removable partial dentures (RPDs) has proven to be clinically suitable and successful for decades to improve the quality of life of partially edentulous patients. ${ }^{1,2}$ Traditional clasp-retained dentures are widely used for partially edentulous patients, particularly in Korea, in accordance with the health insurance policies for patients above 65 years of age. However, in those with few remaining teeth or with periodontally 
compromised abutment teeth, the prognosis of the clasp-retained dentures, as well as the abutment teeth, can be unfavorable. ${ }^{3}$ In such cases, double crown removable partial dentures can be a treatment alternative to traditional clasp-retained dentures, as they provide secondary splinting for the remaining abutment teeth and transfer the chewing forces along the long axis of the abutment tooth, which preserves the integrity of the periodontal ligament. ${ }^{4,5}$ Thus, it can be used in patients with a poor prognosis for clasp-retained partial dentures. Additionally, numerous studies have reported high comfort and favorable long-term survival rates of patients treated with double crown removable partial dentures. ${ }^{6}$

The double crown design concepts can be categorized based on their retention mechanism into conical, telescopic, and electroformed double crowns. ${ }^{7}$ For retention, the conical crowns use a wedging effect, while the telescopic crowns use the friction of the opposing surfaces of the inner and outer crowns, ${ }^{8}$ and the electroformed crowns get their retentive forces from the hydraulic impact and adhesion. ${ }^{9}$ In telescopic double crowns, a clearance fit between the inner and outer crowns provides no retentive force, in which case, additional prefabricated elements are needed for retention and this double crown system is called hybrid telescopic crown with a friction pin. ${ }^{10}$ For the following case, hybrid telescopic double crowns with friction pin method were used. A channel between the inner and outer crowns is prepared using spark erosion, and a pin is inserted for retention. This system has several advantages. First, because the friction pin can be applied in a small area, the size of the inner crown can be minimal, which allows the outer crown to be more aesthetically formed. Second, the retentive force can be modified by the friction pin, which can be easily repaired when fractured..$^{10,11}$

Several kinds of materials, such as gold alloys, Cr-Co metal alloys, titanium, and zirconia, can be used for the inner and outer crowns of double crown dentures. ${ }^{12}$ The different types of materials, as well as retention, can result in different kinds of wear, which can lead to technical problems in denture. ${ }^{7}$ Studies have shown that the major technical problem for double crown dentures is loss of retention, particularly for double crowns that use gold alloy for the inner and outer crowns, in which case the retention loss appeared to occur more often. ${ }^{13,14}$ Thus, Cr-Co metal alloys have been used for the inner and outer crowns of the new tooth-implant-supported double crown dentures.

This case report describes a patient who lost the abutment teeth of a double-crown denture, and the prognosis of the remaining natural teeth was questionable. Without extracting the remaining abutment teeth and using its original inner crown, additional implants were strategically placed to form polygonal support. ${ }^{15}$ The new tooth-implant-supported hybrid telescopic double crown denture with a friction pin was fabricated and was both functionally and esthetically satisfactory. 


\section{II . Case Report}

A 62-year-old male patient visited our clinic complaining of increased tooth mobility, gingival recession, and difficulty in mastication in October 2004 (Fig. 1). After clinical and radiographic examination, the maxillary and mandibular teeth with poor prognosis were extracted, and intensive periodontal treatment was performed on the remaining dentition. Through detailed prosthetic consultation with the patient, a conus double-crown-retained RPD using gold alloy for the inner and outer crowns was selected for the maxilla, while a fixed prosthetic denture was chosen for the mandible. The maxillary left lateral incisor, first premolar, first molar, and second molar were used as abutments for the conus double-crown denture. The prostheses were delivered in February 2005 (Fig. 2).

During follow-up appointments, the patient reported a gradual retention loss of the maxillary conus double-crown denture. After 12 years, due to loss of retention, the patient needed a new prosthesis. Clinical and radiographic examinations revealed a severe bone loss in tooth \#26 with grade 3 mobility (Fig. 3).

Tooth \#26 was evaluated as non-restorable and extracted, and three implants were placed on the right side of the maxilla to allow polygonal support of the new denture. The remaining abutment teeth had no

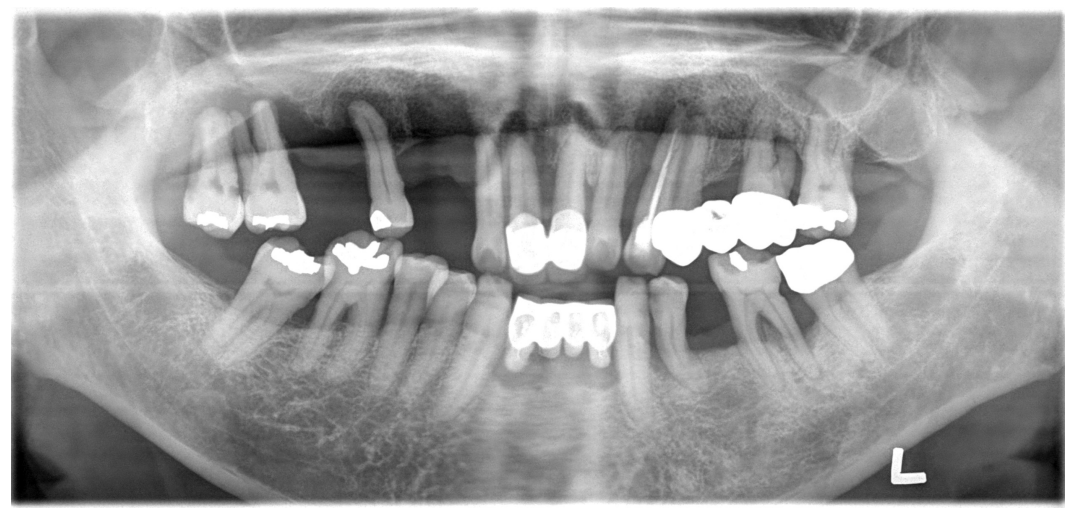

Fig. 1. Initial panoramic radiograph of the patient.

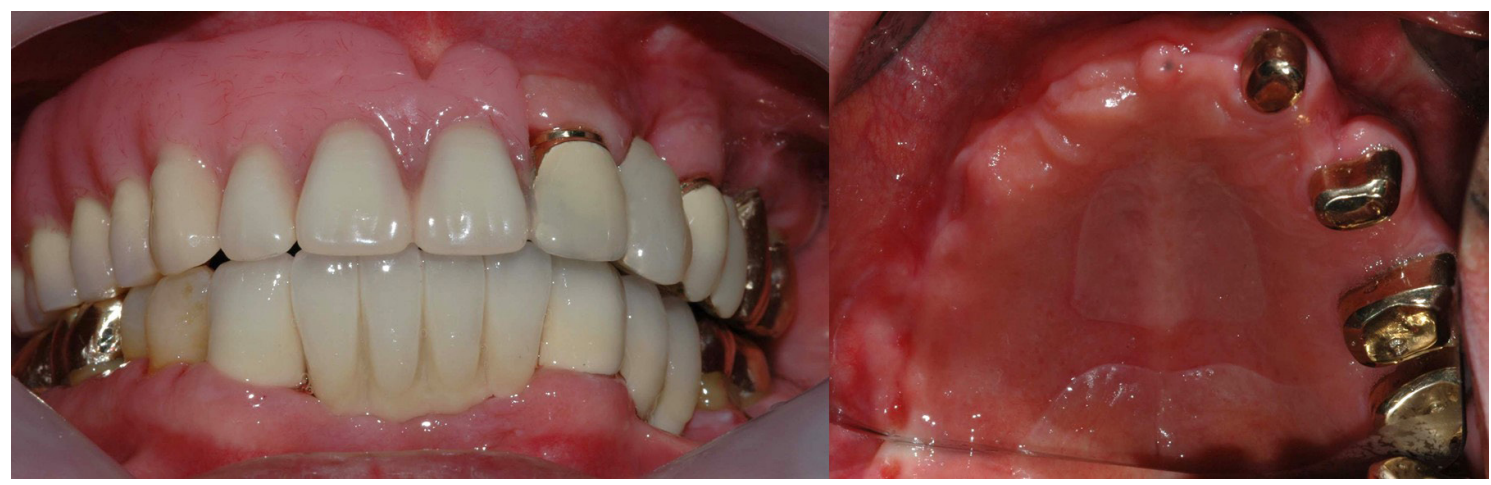

Fig. 2. Frontal and occlusal view of the initial prosthesis. 
clinical problems; therefore, the original inner gold crown was planned for use. The original maxillary denture was relined using a soft reliner (COE-COMFORT ${ }^{\mathrm{TM}}$; GC America, IL, USA) and used as a provisional denture (Fig. 4). The definitive restoration was planned as a hybrid telescopic double crown denture with the friction pin method using the remaining inner gold crowns and an additional three implants for abutments.

Three months later, after confirmation of implant osseointegration, prosthetic treatment was initiated. A polyether impression (Aquasil Ultra LV; Dentsply, Milford, DE, USA) of the maxillary implants and original teeth with the gold inner crowns was made using a custom tray. The impression was poured using type IV dental gypsum (GC Fujirock EP; GC Europe NV, Leuven, Belgium).

A recording base was fabricated on the master cast for vertical dimension determination and centric relation records. The occlusal rim was adjusted intraorally, and a centric record was prepared using a bite registration material (Cresil bite; Creden, Daegu, Korea) (Fig. 5).

For the original inner gold crowns, a clearance fit was achieved between the outer crowns, which provided stability and support for the definitive prosthesis; however, not retention. In contrast, for each of the three implants that were additionally placed, a channel measuring approximately $0.8 \mathrm{~mm}$ was designed using spark erosion for a friction pin between the inner and outer crown, either mesially or distally. A friction pin was placed in this channel, and retention was achieved. The axial convergence of

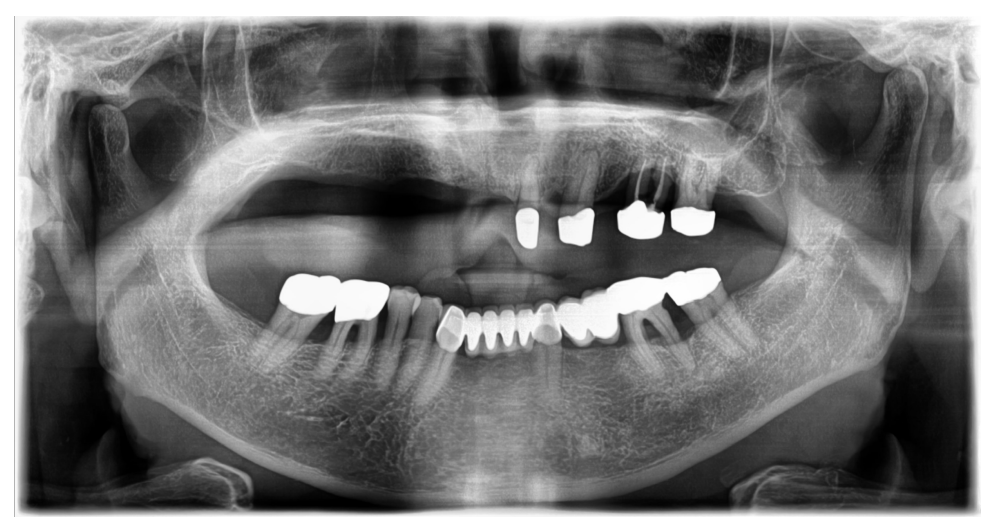

Fig. 3. Panoramic radiograph 12 years after delivery of the initial prosthesis.

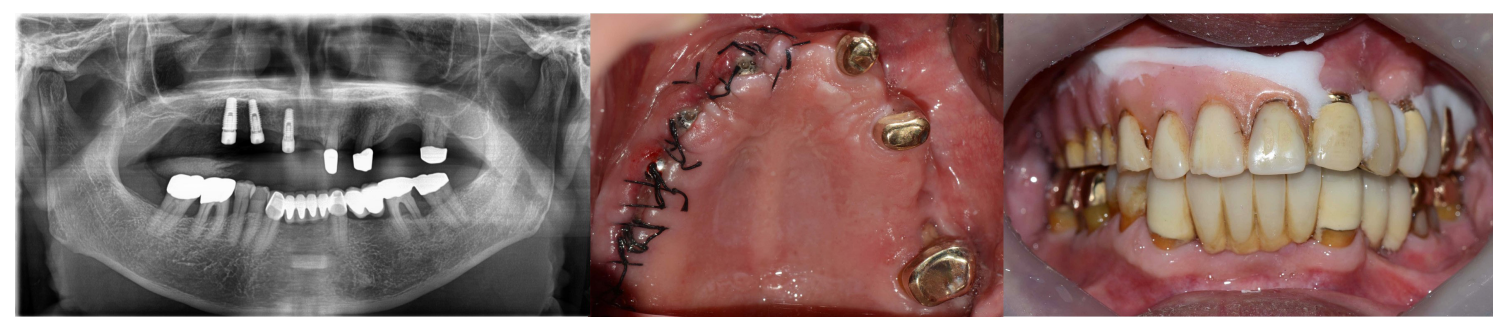

Fig. 4. The original maxillary denture used as a temporary denture after relining. 
the implant's inner crowns was set to $2^{\circ}$, and a chamfer margin was designed to prevent movement between the inner and outer crowns.

The outer crowns and the metal framework were fabricated using a $\mathrm{Co}-\mathrm{Cr}$ alloy (VeraBond 2V; Aalbadent, Fairfield, CT, USA). Wax-up was performed on the outer crowns, and the artificial teeth

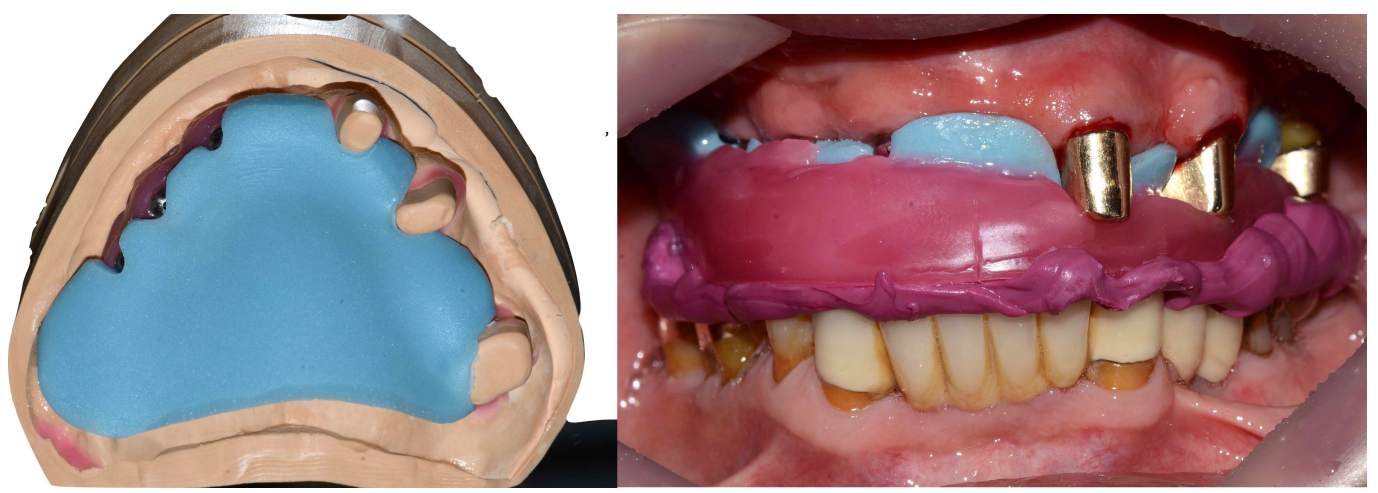

Fig. 5. Centric relation record using recording base fabricated on the master cast.

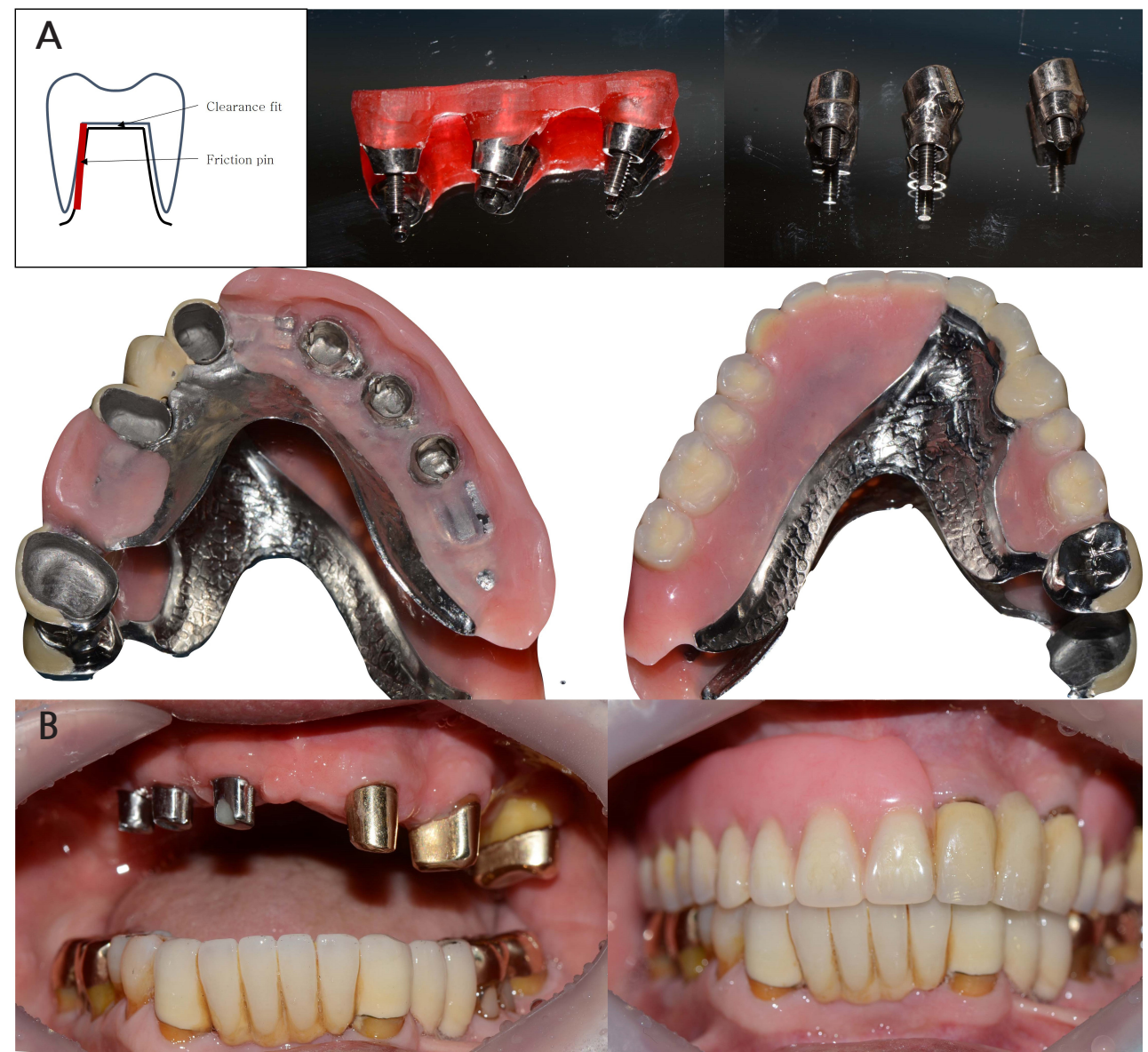

Fig. 6. (A) Schematic diagram of hybrid telescopic double crown with friction pin, and definitive prosthesis before delivery. (B) Frontal view of the definitive prosthesis. 
were arranged according to the determined vertical dimension and centric relation. Esthetic and centric relations were confirmed during the wax try-in.

After denture curing and resin facing the outer crowns, the definitive prostheses were completed and delivered to the patient (Fig. 6). The patient was overall satisfied, particularly with the retention of the removable appliance. The patient was on a regular follow-up schedule for a year after the delivery of the definitive prosthesis. The prosthesis, abutment teeth, and implants were in good condition, with no other complications.

\section{Discussion}

Clinicians are often required to treat elderly patients with reduced dentition as the average life span has increased. Some patients cannot be treated using fixed prostheses due to an insufficient amount of available bone, general diseases, or inability to afford the treatment. Thus, treatment using RPDs is common. In cases with a few remaining teeth, it is challenging to design clasp-retained RPDs, and the prognosis of the abutment teeth can be questionable. In these situations, reconstruction using doublecrown-retained RPDs can be favorably used, particularly when the few remaining teeth are in unfavorable positions for other prosthetic reconstructions. Recent reports have also shown that double-crownretained RPDs demonstrate better clinical performance than clasp-retained RPDs. ${ }^{16}$ It is also more esthetic than clasp-retained RPDs as it completely covers the abutments.

In this clinical report, the patient was originally treated with a conus-double-crown-retained RPD using gold alloy for the inner and outer crowns. After 12 years, the retention loss due to loss of one abutment tooth required the fabrication of a new prosthesis; therefore, a tooth-implant combined hybrid telescopic double crown denture with the friction pin method was planned. The remaining three teeth with gold inner crowns were used as abutments without any modifications, and they were designed to have a clearance fit between the inner gold and outer crowns, which provided support and stability to the definitive prosthesis. To stabilize the new prosthesis, three additional implants were placed on the right side of the maxilla, and for retention, a friction pin was placed between each implant abutment and the outer crown of the new double-crown-retained RPD. ${ }^{11}$

As shown in this case, double crown-retained RPDs with inner and outer crowns fabricated with a precious metal have the significant problem of diminished retention of the prostheses after long-term use due to wearing between the inner and outer crowns, leading to less friction. ${ }^{13}$ The hybrid telescopic double crown denture with friction pin method uses non-precious metal alloys, which have several advantageous properties compared to precious metals such as lower thermal conductivity, lower cost, and higher strength. ${ }^{10}$ 
In the past, if a patient had only a few teeth left in their highly reduced dentition, the remaining teeth were routinely extracted for implant-retained prosthetic rehabilitation. However, tooth-implant combined double-crown-retained dentures can provide more favorable clinical outcomes without extraction of the remaining teeth. For example, Krennmair et al. ${ }^{17}$ reported $100 \%$ survival of the teeth, implants, and prostheses after an average of 38 months, while a study on fixed and removable partial dentures supported by implants and teeth by Nickenig et al. ${ }^{18}$ showed that less than $5 \%$ of the biological or technical complications occurred in implant abutments after 10 years. The higher overall cumulative survival rate of tooth-implant combined double-crown-retained dentures compared to double-crown-retained RPDs with only natural teeth may be due to the secondary splinting effect of the double-crown-retained RPDs. During mastication, osseointegrated implants can distribute their stresses to the surrounding cortical bone, ${ }^{19}$ and by secondary splinting, the abutment teeth and their supportive tissues receive reduced stress. Thus, implant abutments of tooth-implant-associated double-crown-retained RPDs may protect the abutment teeth by relieving functional loading. ${ }^{20}$

In this case, three teeth and three implants were used as abutments for the definitive prosthesis. Fortunately, the number and position of abutments, in this case, were favorable compared to other patients. In many cases, there are a limited number of abutments, and the position might be linear or triangular, which leads to unfavorable support of the double-crown-retained denture. ${ }^{15}$ In these situations, the role of tissue support is more important than in cases with polygonal or quadrangular abutment support. To achieve adequate tissue support, the impression surface must be designed and made with a functional impression and sufficient surface. Additionally, since alveolar ridge resorption occurs with time, periodic relining should be performed whenever the denture seems to be ill-fitting.

Treatment of tooth-implant-associated hybrid telescopic double crown dentures with the friction pin method requires precise and special skills of both the clinician and the dental technician, and it may lead to a higher cost of the prosthesis. Additionally, selection of the remaining teeth as abutments and strategic planning of the number and location of implants is critical to the success of this type of prosthetic treatment. Nevertheless, this clinical report indicates that the additional placement of implants and prosthetic restoration by tooth-implant-associated hybrid telescopic double crown denture with the friction pin method can be successful in cases where double-crown-retained RPDs failed due to loss of some abutment teeth.

\section{Conclusion}

This clinical report shows that a patient who lost some abutment teeth of the original double crown denture and poor prognosis of the remaining natural abutment teeth. Through strategically placed 
additional implants, a new tooth-implant-supported hybrid telescopic double-crown denture with a friction pin was satisfactory for the patient. When clinicians plan prosthetics for patients with few remaining teeth in isolation, additional implant placement and tooth-implant-associated hybrid telescopic double crown dentures using the friction pin method may offer a treatment alternative that has a better prognosis than conventional RPDs.

\section{References}

1. Owall G, Bieniek KW, Spiekermann H. Removable partial denture production in western germany. Quintessence Int 1995;26:621-7.

2. Inukai M, Baba K, John MT, Igarashi Y. Does removable partial denture quality affect individuals' oral health? J Dent Res 2008;87:736-9.

3. Phoenix RD, Cagna DR, DeFreest CF. Stewart KL. Stewart's clinical removable partial prosthodontics. 4th ed. Hanover Park: Quintessence Pub.; 2008. p. 185-8.

4. Wenz HJ, Hertrampf K, Lehmann KM. Clinical longevity of removable partial dentures retained by telescopic crowns: outcome of the double crown with clearance fit. Int J Prosthodont 2001;14:20713 .

5. Widbom T, Löfquist L, Widbom C, Söderfeldt B, Kronström M. Tooth-supported telescopic crownretained dentures: an up to 9-year retrospective clinical follow-up study. Int J Prosthodont 2004;17:2934.

6. Koller B, Att W, Strub JR. Survival rates of teeth, implants, and double crown-retained removable dental prostheses: a systematic literature review. Int J Prosthodont 2011;24:109-17.

7. Engels J, Schubert O, Güth JF, Hoffmann M, Jauernig C, Erdelt K, et al. Wear behavior of different double-crown systems. Clin Oral Investig 2013;17:503-10.

8. Koller B, Att W, Strub JR. Survival rates of teeth, implants, and double crown-retained removable dental prostheses: a systematic literature review. Int J Prosthodont 2011;24:109-17.

9. Beuer F, Edelhoff D, Gernet W, Naumann M. Parameters affecting retentive force of electroformed double-crown systems. Clin Oral Investig 2010;14:129-35.

10. Weber H, Frank G. Spark erosion procedure: a method for extensive combined fixed and removable prosthodontic care. J Prosthet Dent 1993;69:222-7.

11. Jo LJ. Spark erosion process: an overview. J Dent Implant 2011;1:2-6.

12. Verma R, Joda T, Bragger U, Wittneben JG. A systematic review of the clinical performance of tooth-retained and implant-retained double crown prostheses with a follow-up of $\geq 3$ years. $\mathrm{J}$ Prosthodont 2013;22:2-12.

13. Ohkawa S, Okane H, Nagasawa T, Tsuru H. Changes in retention of various telescope crown assemblies over long-term use. J Prosthet Dent 1990;64:153-8.

14. Güngör MA, Artunç C, Sonugelen M. Parameters affecting retentive force of conus crowns. J Oral Rehabil 2004;31:271-7.

15. Mitrani R, Brudvik JS, Phillips KM. Posterior implants for distal extension removable prostheses: a retrospective study. Int J Periodontics Restorative Dent 2003;23:353-9. 
16. Ishida K, Nogawa T, Takayama Y, Saito M, Yokoyama A. Prognosis of double crown-retained removable dental prostheses compared with clasp-retained removable dental prostheses: a retrospective study. J Prosthodont Res 2017;61:268-75.

17. Krennmair G, Krainhöfner M, Waldenberger O, Piehslinger E. Dental implants as strategic supplementary abutments for implant-tooth-supported telescopic crown-retained maxillary dentures: a retrospective follow-up study for up to 9 years. Int J Prosthodont 2007;20:617-22.

18. Nickenig HJ, Spiekermann H, Wichmann M, Andreas SK, Eitner S. Survival and complication rates of combined tooth-implant-supported fixed and removable partial dentures. Int $\mathrm{J}$ Prosthodont 2008;21:131-7.

19. Lin CL, Wang JC, Chang WJ. Biomechanical interactions in tooth-implant-supported fixed partial dentures with variations in the number of splinted teeth and connector type: a finite element analysis. Clin Oral Implants Res 2008;19:107-17.

20. Chen Y, Wang C, Huang Y, Feng T, Zou H, Fan Y. Biomechanical evaluation of the natural abutment teeth in combined tooth-implant-supported telescopic prostheses: a three-dimensional finite element analysis. Comput Methods Biomech Biomed Engin 2017;20:967-79. 